\title{
Treatment Outcomes for Primary Retromolar Trigone Carcinoma: A Single Institution Experience
}

\author{
Original Investigation \\ Uygar Levent Demir (1D, Halide Öztürk Yanaşma \\ Department of Otolaryngology, Uludağ University School of Medicine, Bursa, Turkey
}

Abstract

ORCID iDs of the authors: U.L.D. 0000-0002-9590-1420 H.Ö.Y. 0000-0001-8544-6674.

Cite this article as: Demir UL, Öztürk Yanaşma H. Treatment Outcomes for Primary Retromolar Trigone Carcinoma: A Single Institution Experience. Turk Arch Otorhinolaryngol 2020; 58(2):87-92.

\section{Corresponding Author:}

Uygar Levent Demir, uygardemir@hotmail.com

Received Date: 29.01 .2020 Accepted Date: 09.04 .2020

Content of this journal is licensed under a Creative Commons Attribution 4.0 International License. Available online at www.turkarchotolaryngol.net
Objective: Retromolar trigone (RMT) is a rare location for oral cavity cancers. RMT cancers are aggressive malignancies that mostly present at an advanced stage. In this study, we aimed to evaluate treatment outcomes in patients who underwent initial radical surgical resection and postoperative radiotherapy or chemoradiotherapy with a diagnosis of primary RMT squamous cell carcinoma in our institution.

Methods: The study included 20 primary RMT tumor patients out of 191 oral cavity cancer cases treated from January 2010 through December 2019. We retrospectively analyzed treatment details, histopathology reports, postoperative clinical course and survival outcomes.

Results: The mean age at presentation was 59.4 years. Eighty percent of all patients were either stage 3 or stage 4 . We performed mandibular resection in 14 patients (70\%) and partial maxillectomy in eight patients (40\%). Nineteen patients (95\%) underwent unilateral neck dissection. The incidence of metastatic cervical lymph node was 13/20 (65\%). Overall survival (OS) and disease-free survival (DFS) rates during follow-up (mean 26.3 months) were $60 \%$ and $75 \%$, respectively. There was statistical significance between presence of multilevel metastatic lymph nodes and OS ( $p=0.013)$. DFS and OS of early stage and advanced stage groups were $100 \%$ vs $75 \%$ and $100 \%$ vs $50 \%$, respectively, with no statistical significance ( $\mathrm{p}=0.189$ and $\mathrm{p}=0.084)$.

Conclusion: The survival of advanced stage RMT cancer is poor despite appropriate treatment. Bone involvement that necessitates resection is common due to the proximity of the tumor to the mandible and the maxilla. Multilevel positive cervical lymph nodes and advanced stage are poor prognostic factors.

Keywords: Oral cavity, cancer, retromolar trigone, surgery, survival

\section{Introduction}

Recently, The American Head \& Neck Surgery Society reported the incidence of oral cavity cancers as 4.3 in 100,000 per year, with squamous cell carcinoma (SCC) being the most common histological type seen in more than $90 \%$ of all cases $(1,2)$. Oral cavity has various subsites, namely, the floor of mouth, the lower gum, the upper gum, the oral tongue, the hard palate, the buccal mucosa and the retromolar trigone (RMT) (3). RMT cancers account for only $1.4 \%$ to $5.6 \%$ of all oral cavity cancers $(4,5)$. While the clinical behavior of these cancers vary depending on the affected subsite, the surgical approaches and the reconstructive techniques used are all unique although the mentioned sites are only few millimeters apart. The RMT is a triangular mucosal fold located posterior to the last mandibular molar and bound laterally by the ramus of the mandible and medially by the temporal crest $(6,7)$. Although primary RMT cancers are rare compared to those of the oral tongue and floor of mouth (3-5), they can invade the mandible, the pterygoid muscles, the masticator space and the infratemporal fossa with perineural and lymphatic spread in early stages, causing a poor prognosis $(6,8,9)$.

There are various modalities used in the treatment of RMT cancers, including surgical resection alone $(6,10)$, radiotherapy (RT) alone (11-13), adjuvant therapies RT or chemoradiotherapy (CRT) fol- 
lowing surgery $(6,8,9,13-15)$ or combined therapy using CRT (16). RMT has a complex anatomy with proximity to many vital structures, and any cancer in this location may possess an aggressive behavior which makes the management of these tumors a real challenge for head and neck surgeons. There is no consensus on the standard management and the prognosis is still poor despite appropriate treatment methods $(3,5,9)$. In the literature review we found no study which addressed the treatment outcomes of RMT cancers in Turkey, except a few case reports (17, 18). In this study, we aimed to present the treatment outcomes and the prognostic factors in patients who underwent initial radical surgical resection and postoperative RT or CRT with the diagnosis of primary SCC of RMT at our institution.

\section{Methods}

In this retrospective study, we reviewed the data of the patients that underwent surgical resection and postoperative adjuvant $\mathrm{RT}$ or CRT as primary treatment with the diagnosis of RMT SCC at the department of Otolaryngology and Head Neck Surgery, Uludağ University. Patients with oral cavity cancers who had secondary RMT involvement or received RT or CRT as primary treatment were excluded. Twenty patients out of 191 oral cavity cancer patients treated from January 2010 through December 2019 were included in the study. The clinical information was retrieved from the patients' medical reports at the digital database of the hospital.

The data obtained from the patients' files were assessed for further analysis and data such as gender, age, presenting symptoms, details of surgical procedure, neck status, reconstruction method, TNM classification and stage (according to $7^{\text {th }}$ edition of the AJCC), histopathological findings, postoperative clinical course, oral intake and removal of tracheotomy tube, adjuvant treatment, complications, follow-up duration, recurrence and disease-free survival (DFS) and overall survival (OS) rates were recorded. To assess clinical TNM staging, we requested the computed tomography $(\mathrm{CT})$ of neck, thorax and abdomen in all patients before surgery. Finally, survival rates and clinical variables were analyzed to explore any possible correlations. We had obtained written informed consent from the patients at their initial visit prior to treatment, and Ethical Committee of Uludağ University School of Medicine approved this retrospective study (December 2019/2019-21/11).

\section{Statistical Analysis}

Statistical analysis was performed using the Statistical Package for Social Sciences version 21.0 (IBM SPSS Corp.; Armonk, NY, USA). Normal distribution of the data was assessed by the Shapiro Wilks Test. Since the data were normally distributed,

\section{Main Points}

- Retromolar trigone cancers are rare but highly aggressive malignancies that mostly present at an advanced stage.

- Advanced tumor stage and existence of metastatic cervical lymph nodes had negative impacts on survival.

- Surgery with negative surgical margins followed by RT or CRT shows favorable outcomes at early stages. descriptive statistics are given as mean, standard deviation. The descriptive analysis of the included cases and the association between independent variables and survival rates were calculated by the Fischer's exact and Fisher Freeman Halton tests. Survival analysis was assessed by the log-rank test and carried out by $\mathrm{Ka}^{-}$ plan-Meier curves based on OS and DFS. A significance value of 0.05 was used for all tests.

\section{Results}

There were 20 patients ( 15 males and five females) that had undergone surgery for primary RMT cancer. Thus, RMT cancers constituted approximately 10\% (20/191) of all oral cavity cancer cases treated at our clinic in the last decade. The mean age of study population was 59.4 years (range, $27-76$ years). The mean follow-up time was 26.3 months (range, 1-82 months). Four patients were in the early stage and 16 patients in the late stages according to histopathological reports. No patients had distant metastasis diagnosed by preoperative radiological evaluation. The clinical stages remained identical with pathological stages in 18 patients (90\%) but had progressed to an advanced stage in two patients after final histopathological examinations. Pathological TNM stages of the patients are shown in Table 1.

The extent of resections and neck dissections were determined according to tumor size, depth, location, invasion and lymph node status with the guidance of neck CT scans. In all patients the surgical margins were evaluated by frozen section, and resection field was advanced until negative margins were achieved. The preferred approach to primary tumor was lip split incision with composite resection in 14 patients, en-bloc tumor resection with lip split incision in four patients and transoral resection in two patients. We performed segmental mandibulectomy in eight patients, marginal mandibulectomy in five patients, hemimandibulectomy in one patient and no mandibular resection in six patients. Partial maxillectomy was required in eight patients. One patient had only partial maxillectomy. Among these patients eight had bone involvement confirmed by histopathological examination. In one patient (T1 stage) we did not perform neck dissection. All other patients underwent unilateral neck dissection and the distribution was level 1-3 dissection in two patients, level 1-4 dissection in nine patients, level 1-5 dissection in eight patients. None of the patients needed contralateral neck dissection (Table 2).

There was no metastatic lymph node in seven (35\%) patients. Positive lymph node was found at single level in six patients and multilevel positivity was reported in seven patients. Four patients (20\%) had occult lymph node metastasis that was not clinically suspected before the surgery and underwent elective neck dissection. In all patients, final pathology report confirmed the margin-negative resection analysis similar to the frozen section evaluation. The grade of the tumor was well-differentiated in three, moderately differentiated in 11 , poorly differentiated in two and not available in four patients. Perineural infiltration was present in seven patients. Reconstruction of the defect was achieved by primary closure using skin grafts in five patients, pectoralis major musculocutaneous flap in ten patients, an- 
terolateral thigh free musculocutaneous flap in three patients, sternocleidomastoid muscle flap in one patient and temporalis muscle flap with fascia in one patient (Table 2). In only two patients, mandibular reconstruction was performed using titanium plates. In three patients, we observed local soft tissue infections that were resolved by intravenous antibiotic treatment. Eight patients underwent minor reoperations due to flap dehiscence, necrosis or for scar removal.

The mean time to oral intake and removal of nasogastric feeding tube was 19.6 days (range, 6-52 days). In two patients, the gastrostomy tube was placed due to inadequate oral intake. Tracheotomy tubes were removed in mean 14 days (range, 2-25 days). One patient could be decannulated only after hyoid suspension was performed on the $25^{\text {th }}$ day.

After surgical intervention, adjuvant RT or CRT were given to high risk patients: Nine received adjuvant radiotherapy alone, eight received adjuvant CRT. Three early-stage patients did not receive adjuvant therapy. Adjuvant therapy was given to advanced-stage patients who had cervical lymph node metastasis with extracapsular extension, surgical margin positivity, presence of perineural or perivascular infiltration or presence of bone invasion. Recurrence was not seen in 13 (65\%) out of 20 patients during the follow-up period. Three patients had locoregional recurrence and four had distant metastasis. Of the patients with local recurrence, two underwent salvage surgery and one received chemotherapy. In all patients with distant metastasis, the lung was the site of involvement and they received chemotherapy. Two of these patients also had liver and intraabdominal lymph node involvements. Five patients died from the

Table 1. Distribution of patients by $\mathrm{T}$ and $\mathrm{N}$ classification based on the $7^{\text {th }}$ edition of the AJCC manual

\begin{tabular}{|c|c|c|c|c|c|}
\hline \multirow[b]{2}{*}{$\mathrm{N}$ classification } & \multicolumn{4}{|c|}{$\mathrm{T}$ classification } & \multirow[b]{2}{*}{ Tota } \\
\hline & $\mathrm{T} 1$ & $\mathrm{~T} 2$ & T3 & $\mathrm{T} 4$ & \\
\hline N0 & 1 & 3 & 0 & 3 & 7 \\
\hline N1 & 1 & 0 & 2 & 1 & 4 \\
\hline N2 & 1 & 1 & 2 & 2 & 6 \\
\hline N3 & 0 & 0 & 1 & 2 & 3 \\
\hline Total & 3 & 4 & 5 & 8 & 20 \\
\hline
\end{tabular}

recurrence of the primary disease or associated conditions, but other three patients died from unrelated medical causes. As a result, the rate of DFS was found $75 \%$ (15/20) and that of OS was found $\% 60(12 / 20)$ in our study population for an average follow-up duration of 26 months (Figure 1).

Finally, we evaluated the possible correlation between DFS and OS and risk factors such as tumor grade, perineural infiltration, $\mathrm{T}$ stage, $\mathrm{N}$ status, extent of neck dissection, multilevel positive lymph nodes. Tumor grade, perineural infiltration and $\mathrm{T}$ stage did not show any correlation with survival rates. DFS ratio was 6/7 (85\%) in patients with negative lymph node and 9/13 (69\%) in those with positive lymph node. OS ratio was $6 / 7(85 \%)$ in patients with negative lymph node and 6/13 (46\%) in those with positive lymph node. There was a significant difference only between the presence of multilevel metastatic lymph nodes and OS $(p=0.013)$, which revealed poorer prognosis in these patients. As we classified patients into two groups as early stage (stages 1 and 2) ( $n=4)$ and advanced stage (stages 3 and 4$)(n=16)$ according to the TNM staging, the final comparison was made between DFS and OS in these subgroups. All four patients in the early stage were alive (OS: 100\%) on the closing day of the study, but unfortunately eight of the advanced stage patients 50\% (8/16) died in the follow-up period. Despite the manifest difference between the DFS (100\% vs $75 \%)$ and the OS (100\% vs $50 \%)$ rates of the early stage and advanced stage groups, the $\mathrm{p}$ value did not reach significance $(\mathrm{p}=0.189$ and $\mathrm{p}=0.084)$ (Figure 2$)$. We attributed this insignificance to the low number of the study population.

\section{Discussion}

Although primary SCC of the RMT is rare, these tumors generally present at an advanced stage because of their unique anatomical location $(8,9)$. Because they are seated at the junction of the mandible, the alveolar arcus, the tonsillar pillar, the buccal space, the masticator space, the base of the tongue or the palate, they may easily invade all these structures simultaneously at an early period (14). More importantly, since there is only a thin mucosal barrier between the RMT tumor border and the underlying bone, bone involvement is a common feature of these tumors that upstage even small lesions to T4 (10). All these factors may influence colleagues to underestimate the real extent of the tumor in clinical examination and require further imaging techniques to make true diagnosis. Tumor size is an important determinant for $\mathrm{T}$ stage assessment in oral cavity cancers; how-

Table 2. Surgical procedures and reconstruction techniques in $\mathrm{T}$ stages

\begin{tabular}{|c|c|c|c|c|c|c|c|c|c|c|c|c|c|c|}
\hline \multirow{2}{*}{$\begin{array}{l}\mathrm{T} \\
\text { classification }\end{array}$} & \multicolumn{5}{|c|}{ Bone resection } & \multicolumn{4}{|c|}{ Neck dissection (level) } & \multicolumn{5}{|c|}{ Reconstruction } \\
\hline & none & $\mathrm{mm}$ & $\mathrm{sm}$ & $\mathrm{hm}$ & $\mathrm{mx}$ & I-III & I-IV & $\mathrm{I}-\mathrm{V}$ & none & PS & PM & FF & TM & SCM \\
\hline $\mathrm{T} 1$ (n: 3) & 2 & 1 & 0 & 0 & 0 & 1 & 0 & 1 & 1 & 2 & 0 & 1 & 0 & 0 \\
\hline $\mathrm{T} 2$ (n: 4) & 2 & 0 & 2 & 0 & 0 & 1 & 2 & 1 & 0 & 2 & 2 & 0 & 0 & 0 \\
\hline T3 (n: 5) & 0 & 4 & 1 & 0 & 4 & 0 & 3 & 2 & 0 & 1 & 2 & 1 & 0 & 1 \\
\hline $\mathrm{T} 4(\mathrm{n}: 8)$ & 1 & 0 & 5 & 1 & 4 & 0 & 4 & 4 & 0 & 0 & 6 & 1 & 1 & 0 \\
\hline Total & 5 & 5 & 8 & 1 & 8 & 2 & 9 & 8 & 1 & 5 & 10 & 3 & 1 & 1 \\
\hline
\end{tabular}

mm: marginal mandibulectomy; sm: segmental mandibulectomy; hm: hemimandibulectomy; mx: partial maxillectomy; PS: primary closure with skin graft; PM: pectoralis major; musculocutaneous flap; FF: free musculocutaneous flap; TM: temporalis muscle flap; SCM: sternocleidomastoid muscle flap 

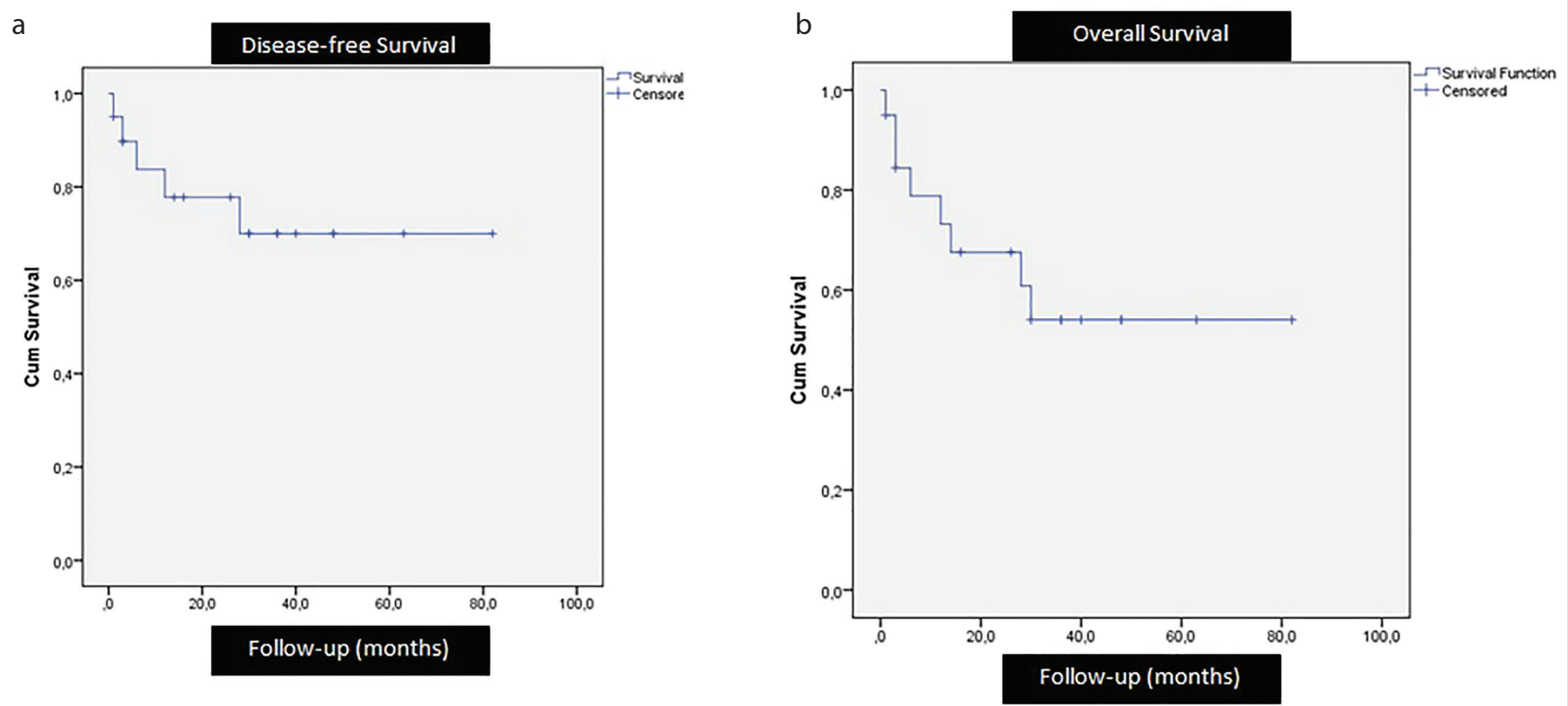

Figure 1.a, b. (a) Kaplan-Meier estimates of DFS and (b) OS rates for all patients. The rate of DFS was $75 \%$ and OS was $60 \%$

a

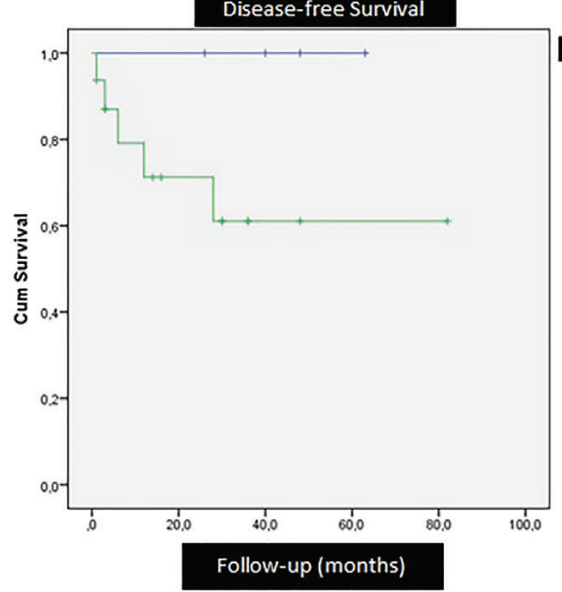

b
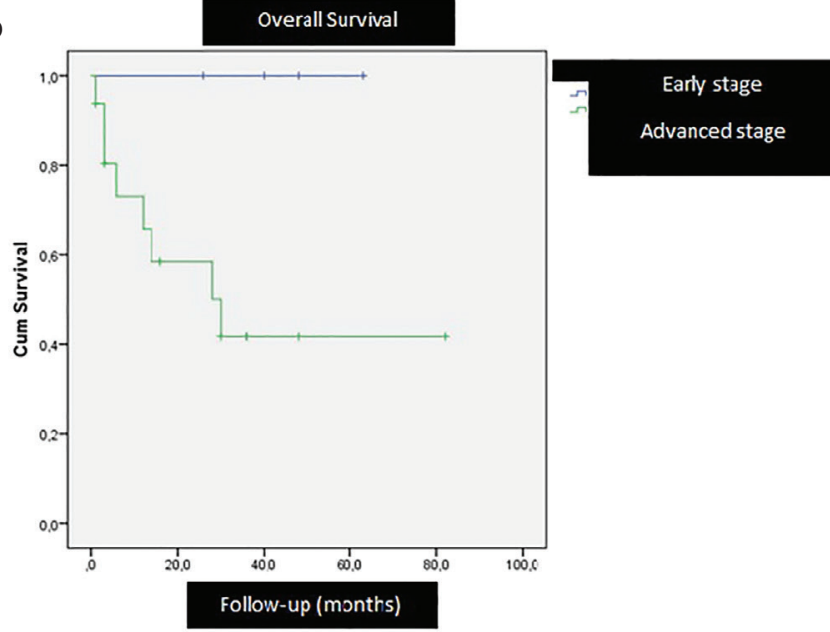

Figure 2. a, b. (a) Kaplan-Meier estimates of DFS and (b) OS rates for early stage and advanced stage patients. The DFS rates for early vs advanced stage patients were $100 \%$ vs $75 \%$, respectively, and OS rates for early vs advances stage patients were $100 \%$ vs $50 \%$, respectively

ever, it is not predictive for the depth of invasion and prognosis, especially in RMT cancers.

Unfortunately, most of the RMT tumors reported in the literature were advanced at presentation. Rizvi et al. (9), evaluating the outcomes of primary SCC of the RMT in a cohort study, reported that nearly $56 \%$ of the patients were at an advanced stage (stages 3 and 4). Similarly, the advanced stage patients constituted $62 \%$ of all patients treated for RMT cancer in the study of Hao et al. (14). Hitchcock et al. (13) analyzed the treatment outcomes of 110 patients who received radiotherapy alone or RT combined with surgery. They pointed out that almost $72 \%$ of their study group was at an advanced stage. Bayman et al. (12) conducted a study with a selective group of RMT cancer patients who were not suitable for surgery and showed no evidence of metastatic disease. These patients received definitive radiotherapy for treatment. The authors reported a 49\% rate of advanced stage patients even in this specific population. In the study of Mendenhall et al. (15), 72 of the patients (73\%) were at stage 3 or 4 among the 99 patients treated for RMT cancer either with RT alone or RT combined with surgery. In a study from Japan (6), patients at stages 3 and 4 accounted for almost $69 \%$ of all the treated RMT cancer cases. In our study, 16 patients (80\%) were at an advanced stage, a finding consistent with the current literature pointing that patients with RMT cancer either typically present to clinics at an advanced stage or are reported to be at an advanced stage by final histopathological examination results.

The impact of the stage on survival was evaluated in the previous studies. Farhood et al. (3) aimed to assess the mortality rates in 
oral cavity carcinomas based on subsites by using Surveillance, Epidemiology and End Results (SEER) program 9 database. They concluded that cause-specific mortality increased by stage and by not receiving surgery and radiation despite any certain subsite. Rizvi et al. (9) reported an OS rate of $38 \%$ at the end of 36-month of follow-up and mentioned that advanced stage showed significantly $(\mathrm{p}<0.001)$ bad impact on survival. Nishi et al. (6) pointed out that the rate of 3-year survival was $59.8 \%$ and DFS rate was $59.7 \%$ in their study population, and survival rates decreased with stage, but without significance; stage $1-100 \%$, stage $2-80 \%$, stage $3-40 \%$ and stage $4-49.2 \%$. In their study which evaluated the outcomes of radical surgery and adjuvant radiotherapy in patients with locally advanced RMT cancer Deo et al. (8) found OS and DFS rates as 71\% and 64\%, respectively. Similarly, Hao et al. (14) reported 5-year survival rate of $60.6 \%$ in all stages together and that this rate dramatically decreased to $43.6 \%$ in stage 4 patients, especially in those with masticator space and/or infratemporal fossa involvement. In our study, we also found that DFS and OS were much lower for patients at advanced stage compared to early stage without significance. We did not find a significant $\mathrm{p}$ value between groups despite big survival difference. This finding may be related with the small sample size in this study.

The prognosis in patients with RMT cancer also greatly depends on the status of the cervical lymph node, as well as the stage of the tumor. Nishi et al. (6) reported that 19 of their 39 patients (48.7\%) were found to have lymph node metastatic disease in histopathologic evaluation. In addition, they showed that there was significant $(\mathrm{p}=0.0001)$ difference at 3 -year survival rates between patients with $(n=19)$ or without $(n=20)$ pathologically positive cervical lymph node; $24 \%$ vs $86.4 \%$, respectively. In the study of Deo et al. (8), cervical lymph node involvement rate was reported as $50 \%$ (21/42). The distribution of positive lymph nodes was only level I in $31 \%$ of the patients (13/42) and level I-II in $19 \%$ of the patients (8/42). The 3 -year DFS rates in patients with pathological node positive and negative were $61 \%$ and $92 \%$, respectively. In a large cohort study using SEER database Rizvi et al. (9) found the incidence of regional node metastasis in RMT cancer as $39.5 \%$ and significant correlation of nodal disease with both OS and DFS. In our study population, $65 \%(13 / 20)$ of the patients were found to have positive nodal metastasis in histopathologic examination. These positive nodes were found at level I in four patients, at level II in 11 patients, at level III in three patients and at level IV in three patients. Similar to the literature, we also found that the rate of OS patients with positive lymph nodes was lower compared to the patients with negative lymph node; $46 \%$ vs $85 \%$, respectively. In seven patients there was multilevel involvement of lymph nodes. We found a significant correlation between the multilevel cervical node positivity and OS ( $\mathrm{p}=0.013)$. Those patients with multilevel metastatic lymph node involvement showed worse prognosis.

Tumor grade has also been evaluated as a risk factor for survival in RMT cancer patients. The prognosis of patients with poorly differentiated oral cavity cancer was found worse in a cohort study of Farhood et al. (3). The majority of patients (76.2\%) with
RMT cancer had well-differentiated tumors in the study of Deo et al. (8), but the authors did not mention its impact on survival. Nishi et al. (6) found that most of RMT cancer patients showed moderately differentiated carcinoma in histopathologic evaluation. The authors did not report about the correlation between prognosis and tumor grade. Similarly, in our study most of the patients had moderately differentiated tumors with no relation to survival. In the literature, mandibular involvement in RMT tumors was reported as high as $14 \%$ to $75 \%(8,19)$. Hao et al. (14) assessed the rate of bone invasion in 50 patients treated for RMT cancer and found that 17 of these patients (34\%) had either mandible or maxilla involvement. In their study, Deo et al. (8) documented the rate of mandibular and maxillary invasion as $47 \%$ and $12 \%$, respectively. In our study, of the 15 patients that underwent either mandibulectomy or maxillectomy or both, bone invasion was confirmed by histopathological examination in eight patients with an overall bone involvement rate of $40 \%$.

The clinical characteristics of RMT tumors are unique compared to other subsites because they possess early invasion to deeper structures, such as the mandible, the masticator space, the lingual nerve or the pterygoid muscles, regardless of superficial tumor size. The diameter does not reflect the actual volume of these tumors. All these properties of RMT tumors lead to increased mortality and low local control rates. Although they constitute a small part of all oral cavity cancers, their prognosis is poor, especially at later stages.

\section{Conclusion}

In this study we found that appropriate surgery with negative surgical margins followed by RT or CRT showed favorable outcomes at early stages. Advanced tumor stage and existence of metastatic cervical lymph nodes had negative impacts on survival. This is the first study from Turkey reporting the treatment outcomes and the prognostic factors in patients with primary RMT cancer. Since the population of the presented study is relatively small, we intend to conduct further multi-center cohort studies to better delineate other prognostic factors and understand the clinical behavior of these rare tumors.

Ethics Committee Approval: Ethics committee approval was received for this study from the Ethics Committee of Uludağ University School of Medicine (Approval Date: December 25, 2019; Approval Number: 2019-21/11).

Informed Consent: Written informed consent was obtained from the patients who participated in this study.

Peer-review: Externally peer-reviewed.

Author Contributions: Concept - U.L.D., H.Ö.Y.; Design - U.L.D., H.Ö.Y.; Supervision - U.L.D., H.Ö.Y.; Materials - U.L.D., H.Ö.Y.; Data Collection and/or Processing - U.L.D., H.Ö.Y.; Analysis and/or Interpretation - U.L.D., H.Ö.Y.; Literature Search - U.L.D., H.Ö.Y.; Writing - U.L.D., H.Ö.Y.; Critical Reviews - U.L.D.

Conflict of Interest: The authors have no conflicts of interest to declare. 
Financial Disclosure: The authors declared that this study has received no financial support.

\section{References}

1. Oral cavity cancer: Professional version - page 3 of 12 . American Head \& Neck Society. Available at: https:/www.ahns.info/ resources/oral-cavity-cancer/3/\#_Toc400976516. Accessed June 21, 2017.

2. World Health Organization classification of tumours: Pathology and genetics of head and neck tumours. Barnes L, Eveson JW, Reichart P, Sidransky D, editors. Vol 9. 3rd ed. Lyon: IARC Publications; 2005.

3. Farhood Z, Simpson M, Ward GM, Walker RJ, Osazuwa-Peters N. Does anatomic subsite influence oral cavity cancer mortality? A SEER database analysis. Laryngoscope 2019; 129: 1400-6. [Crossref]

4. Japan Society for Head and Neck Cancer. Report of head and neck cancer registry of Japan clinical statistic of registered patients, 2011.Jpn J Head Neck Cancer 2013; 39: 15-32.

5. Sowder JC, Cannon RB, Buchmann LO, Hunt JP, Hitchcock $\mathrm{Y}$, Lloyd S, et al. Treatment-related determinants of survival in early-stage (T1-2N0M0) oral cavity cancer: a population-based study. Head Neck 2017; 39: 876-80. [Crossref]

6. Nishi H, Shinozaki T, Tomioka T, Maruo T, Hayashi R. Squamous cell carcinoma of the retromolar trigone: treatment outcomes. $\mathrm{Au}-$ ris Nasus Larynx 2018; 45: 337-42. [Crossref]

7. Athavale SA, Vijaywargia M, Deopujari R, Kotgirwar S. Bony and cadaveric study of retromolar region. People's J Sci Res 2013; 6: 14-8.

8. Deo SV, Shukla NK, Kallianpur AA, Mohanti BK, Thulkar SP. Aggressive multimodality management of locally advanced retromolar trigone tumors. Head Neck 2013; 35: 1269-73. [Crossref]

9. Rizvi ZH, Alonso JE, Kuan EC, St John MA. Treatment outcomes of patients with primary squamous cell carcinoma of the retromolar trigone. Laryngoscope 2018; 128: 2740-4. [Crossref]
10. Horta R, Nascimento R, Silva A, Amarante J. The retromolar trigone: anatomy, cancer treatment modalities, reconstruction, and a classification system.J Craniofac Surg 2016; 27: 1070-6. [Crossref]

11. Ayad T, Gélinas M, Guertin L, Larochelle D, Del Vecchio P, Tabet $\mathrm{JC}$, et al. Retromolar trigone carcinoma treated by primary radiation therapy: an alternative to the primary surgical approach. Arch Otolaryngol Head Neck Surg 2005; 131: 576-82. [Crossref]

12. Bayman NA, Sykes AJ, Bonington S, Blackburn T, Patel M, Swindell R, et al. Primary radiotherapy for carcinoma of the retromolar trigone: a useful alternative to surgery. Clin Oncol (R Coll Radiol) 2010; 22: 119-24. [Crossref]

13. Hitchcock KE, Amdur RJ, Morris CG, Werning JW, Dziegielewski PT, Mendenhall WM. Retromolar trigone squamous cell carcinoma treated with radiotherapy alone or combined with surgery: a 10-year update. Am J Otolaryngol. 2015; 36: 140-5. [Crossref]

14. Hao SP, Tsang NM, Chang KP, Chen CK, Huang SS. Treatment of squamous cell carcinoma of the retromolar trigone. Laryngoscope 2006; 116: 916-20. [Crossref]

15. Mendenhall WM, Morris CG, Amdur RJ, Werning JW, Villaret DB. Retromolar trigone squamous cell carcinoma treated with radiotherapy alone or combined with surgery. Cancer 2005; 103 : 2320-5. [Crossref]

16. Scher ED, Romesser PB, Chen C, Ho F, Wuu Y, Sherman EJ, et al. Definitive chemoradiation for primary oral cavity carcinoma: a single institution experience. Oral Oncol 2015; 51: 709-15. [Crossref]

17. Durmus K, Apuhan T, Ozer E. Transoral robotic surgery for retromolar trigone tumours. Acta Otorhinolaryngol Ital 2013; 33: 4257.

18. Yalçın ED, Süslü N, Avcu N. Retromolar trigone squamous cell carcinoma: case report. J Dent Fac Atatürk Uni 2014; 9: 1-5.

19. Lane AP, Buckmire RA, Mukherji SK, Pillsbury HC 3rd, Meredith SD. Use of computed tomography in the assessment of mandibular invasion in carcinoma of the retromolar trigone. Otolaryngol Head Neck Surg 2010; 122: 673-7. [Crossref] 